TRANSACTIONS OF THE

AMERICAN MATHEMATICAL SOCIETY

Volume 352, Number 1, Pages 363-377

S 0002-9947(99)02178-9

Article electronically published on July 21, 1999

\title{
HANKEL OPERATORS ON BOUNDED ANALYTIC FUNCTIONS
}

\author{
JAMES DUDZIAK, T. W. GAMELIN, AND PAMELA GORKIN
}

\begin{abstract}
For $U$ a domain in the complex plane and $g$ a bounded measurable function on $U$, the generalized Hankel operator $S_{g}$ on $H^{\infty}(U)$ is the operator of multiplication by $g$ followed by projection into $L^{\infty} / H^{\infty}$. Under certain conditions on $U$ we show that either $S_{g}$ is compact or there is an embedded $\ell^{\infty}$ on which $S_{g}$ is bicontinuous. We characterize those $g$ 's for which $S_{g}$ is compact in the case that $U$ is a Behrens roadrunner domain.
\end{abstract}

\section{INTRODUCTION}

Let $X$ be a compact space and let $A$ be a closed unital subalgebra of $C=C(X)$. For fixed $g \in C$, we define the Hankel-type operator $S_{g}$ from $A$ to the quotient Banach space $C / A$ by

$$
S_{g}(f)=g f+A, \quad f \in A .
$$

The algebra $A$ is defined to be tight if the operators $S_{g}$ are weakly compact for all $g \in C$. Cole and Gamelin [8] proved that $A$ is tight if and only if $A^{* *}+C$ is a closed subalgebra of $C^{* *}$. We will say the algebra $A$ is compactly tight if the operators $S_{g}$ are compact for all $g \in C$.

Roughly speaking, an algebra of analytic functions with continuous boundary values is tight whenever the delta-bar problem can be solved with a mild gain in smoothness. In particular, the various algebras $R(K)$ and $A(K)$ associated with rational approximation in the complex plane, and also the algebras $A(D)$ associated with bounded strictly pseudoconvex domains with smooth boundaries in $\mathbb{C}^{n}$, are tight. In fact, the operators solving the delta-bar problem in these examples are compact, so that the operators $S_{g}$ are compact, and these algebras are compactly tight.

These Hankel-type operators appear implicitly in the work of J. Bourgain on the Dunford-Pettis property. Bourgain's work [3] shows that if the operators $S_{g}$ are always completely continuous (weak-null sequences are mapped to norm-null sequences), then $A$ has the Dunford-Pettis property. Thus the algebras described above all have the Dunford-Pettis property (see [7]). The Hankel-type operators are also related via work of Bourgain to the Pelczyński property. Using the work in [4], S. Saccone has shown in [20] that any compactly tight uniform algebra has the Pelczyński property. One consequence of this is that the dual space of a compactly tight uniform algebra is weakly complete.

Now the sets of functions $g \in C$ such that $S_{g}$ is compact, or weakly compact, or completely continuous, are closed subalgebras of $C$ containing $A$. The set of $g \in C$

Received by the editors May 6, 1997.

1991 Mathematics Subject Classification. Primary 46J15, 47B38; Secondary 30D55, 47B05.

(C)1999 American Mathematical Society 
such that $S_{g}$ is completely continuous is called the Bourgain algebra associated with the subalgebra $A$ of $C$.

The algebras of the form $H^{\infty}$ that arise in connection with bounded analytic functions are not tight in general. The problem then is to determine, for a given $g \in L^{\infty}$, when $S_{g}: H^{\infty} \mapsto L^{\infty} / H^{\infty}$ is compact, or weakly compact, or completely continuous. Several papers have appeared identifying the Bourgain algebra for certain $H^{\infty}$-spaces. For the case of the classical algebra $H^{\infty}(d \theta)$ associated with the bounded analytic functions on the open unit disk $\Delta$ in the complex plane, the Bourgain algebra is identified in [5] as $H^{\infty}(d \theta)+C(\partial \Delta)$. Much related work has been done (see for instance [6],[17]). The work with the strongest bearing on our problem is that of K. Izuchi, who obtained in [19] some fairly broad results generalizing many existing descriptions of Bourgain algebras.

We focus here on the algebra $H^{\infty}(U)$ of bounded analytic functions on a fixed bounded open subset $U$ of the complex plane. Let $\sigma$ denote area measure on $U$. We regard $H^{\infty}(U)$ as a subalgebra of $L^{\infty}(\sigma)$, and indeed it is a weak-star closed subalgebra of $L^{\infty}(\sigma)$. For fixed $g \in L^{\infty}(\sigma)$, we consider the Hankel-type operator $S_{g}$ from $H^{\infty}(U)$ to $L^{\infty}(\sigma) / H^{\infty}(U)$ defined by $S_{g}(f)=g f+H^{\infty}(U)$. Our goal is to determine when $S_{g}$ is compact. The set of such $g$, denoted by $B^{\infty}(U)$, is a closed subalgebra of $L^{\infty}(\sigma)$ that contains $H^{\infty}(U)$ and that is invariant under the $T_{\phi}$-operators of rational approximation theory. We show in Section 6 that if every point of $\partial U$ is a peak point for $H^{\infty}(U)$, then $B^{\infty}(U)$ coincides with $\left[H^{\infty}(U)+C(\bar{U})+L_{0}^{\infty}(\sigma)\right]$, the closed linear span of $H^{\infty}(U), C(\bar{U})$, and the space $L_{0}^{\infty}(\sigma)$ of those functions from $L^{\infty}(\sigma)$ that tend to 0 at $\partial U$. In Section 7 we identify $B^{\infty}(U)$ for the class of infinitely connected domains introduced by M. Behrens (see $[1],[2],[21])$. For these domains there is one boundary point that is not a peak point, and $B^{\infty}(U)$ is strictly larger than $\left[H^{\infty}(U)+C(\bar{U})+L_{0}^{\infty}(\sigma)\right]$.

In each of the cases where we are able to obtain complete information, we show that either $S_{g}$ is compact, or else there is a closed complemented subspace of $H^{\infty}(U)$ isomorphic to $\ell^{\infty}$ on which $S_{g}$ is an isomorphism (a bicontinuous operator onto its range). Thus compactness, weak compactness, and complete continuity are equivalent for the operators $S_{g}$ in the cases we have treated. We ask whether the dichotomy holds for all the Hankel operators $S_{g}$, and in particular whether compactness, weak compactness, and complete continuity are always equivalent.

The paper is organized as follows. In Section 2 we provide some background material on when the sum of certain closed subalgebras of $L^{\infty}(\sigma)$ is again a closed subalgebra of $L^{\infty}(\sigma)$, and we show by example that $H^{\infty}(U)+C(\bar{U})$ is not always closed. In Section 3 we treat multiplication operators $M_{g}$, on arbitrary $U$. Sections 4 through 6 are devoted to the Hankel operators $S_{g}$ defined for area measure on $U$, and in Section 7 we treat the Behrens domains. In Section 8 we briefly discuss analogous results for the case of harmonic measure on $\partial U$.

\section{Preliminaries}

We will be interested in when $H^{\infty}(U)+C(\bar{U})$ is closed in $L^{\infty}(\sigma)$. The following criterion is an immediate consequence of the open mapping theorem (cf. Theorem 14.1 of $[8])$.

Lemma 2.1. The sum $M+N$ of two subspaces of a Banach space is closed if and only if there is a constant $c \geq 1$ such that $\operatorname{dist}(x, M \cap N) \leq c \operatorname{dist}(x, N)$ for all $x \in M$. 
We denote $A(U)=H^{\infty}(U) \cap C(\bar{U})$, the algebra of analytic functions on $U$ with continuous boundary values. The weak-star closure $H^{\infty}(A(U), \sigma)$ of $A(U)$ in $L^{\infty}(\sigma)$ is a weak-star closed subalgebra of $H^{\infty}(U)$. The algebra $A(U)$ is a $T$ invariant algebra, invariant under the $T_{\phi}$ operators of rational approximation theory. According to general results on such algebras (Davie's theorem; see [9]), $A(U)$ is pointwise-boundedly dense in $H^{\infty}(A(U), \sigma)$, that is, every function in $H^{\infty}(A(U), \sigma)$ can be approximated pointwise on $U$ by a bounded sequence in $A(U)$. Further, $H^{\infty}(A(U), \sigma)+C(\bar{U})$ is a closed subalgebra of $L^{\infty}(\sigma)$. The formal apparatus of the $T$-invariant algebras applies in part to $H^{\infty}(U)$, and yields the following.

Lemma 2.2. The closure $\left[H^{\infty}(U)+C(\bar{U})\right]$ of the linear span of $H^{\infty}(U)$ and $C(\bar{U})$ is a closed subalgebra of $L^{\infty}(\sigma)$. If $A(U)$ is pointwise boundedly dense in $H^{\infty}(U)$, then $H^{\infty}(U)+C(\bar{U})$ is already a closed subalgebra of $L^{\infty}(\sigma)$.

Proof. Let $\phi$ be a smooth compactly supported function on $\mathbb{C}$, and let $g \in H^{\infty}(U)$ be extended to be zero off $U$. The $T_{\phi}$ operator is defined by

$$
\left(T_{\phi} g\right)(\zeta)=\phi(\zeta) g(\zeta)+\frac{1}{\pi} \iint \frac{g(z)}{z-\zeta} \frac{\partial \phi}{\partial \bar{z}} d x d y .
$$

(See II.1 or VIII.10 of [12], or Section 2 of [15].) Since the convolution of a locally integrable function with a compactly supported bounded Borel function is continuous everywhere, the integral on the right is in $C(\bar{U})$. Since $T_{\phi} g$ is analytic wherever $g$ is, evidently $T_{\phi} g \in H^{\infty}(U)$. Hence $\phi g \in H^{\infty}(U)+C(\bar{U})$. Passing to uniform limits of smooth $\phi$ 's, we obtain the first statement of the lemma. The second statement follows from the remarks preceding the lemma.

It is fairly easy to see that $H^{\infty}(U)+C(\bar{U})$ is not closed when $U$ is a slit disk. By punching out small holes in such a $U$, we are led to the following example (cf. Problem 13.2 in [10]).

Example. There is a connected open subset $U$ of $\mathbb{C}$ such that $U$ is the interior of its closure and such that $H^{\infty}(U)+C(\bar{U})$ is not closed.

Proof. Let $U$ be obtained from the open unit disk $\Delta$ by excising the closed interval $[-1 / 4,1 / 4]$ together with a sequence $\left\{\bar{\Delta}_{n}\right\}$ of disjoint closed disks in the top half of $\Delta$ that accumulate on all of the interval. We assume that the disks have radii $r_{n}$ and distances to the real axis $d_{n}$ satisfying $\sum r_{n} / d_{n}^{2}<\infty$. Since $H^{\infty}(U) \cap C(\bar{U})=A(U)$, it suffices by Lemma 2.1 to find functions $f_{\varepsilon} \in H^{\infty}(U)$ for $\varepsilon>0$ arbitrarily small such that $\operatorname{dist}\left(f_{\varepsilon}, C(\bar{U})\right) \rightarrow 0$ while $\operatorname{dist}\left(f_{\varepsilon}, A(U)\right) \geq c>0$. For this, we let $W_{\varepsilon}$ be the exterior slit domain obtained by deleting the interval $[-\varepsilon,+\varepsilon]$ from the extended complex plane, and we choose $f_{\varepsilon}$ to be the conformal map of $W_{\varepsilon}$ onto the rectangle $\{|x|<1,|y|<\varepsilon\}$ satisfying $f_{\varepsilon}(\infty)=0$ and $f_{\varepsilon}( \pm \varepsilon)= \pm 1$. Then $f_{\varepsilon}$ is symmetric with respect to the real axis, so that the real part $h_{\varepsilon}$ of $f_{\varepsilon}$ is continuous on the slit. Further, the imaginary part of $f_{\varepsilon}$ is bounded by $\varepsilon$, so that $\operatorname{dist}\left(f_{\varepsilon}, C(\bar{U})\right) \leq\left\|f_{\varepsilon}-h_{\varepsilon}\right\| \leq \varepsilon$.

Let $g \in A(U)$. Choose $z_{ \pm}$in the bottom half of $\Delta$ near to $\pm \varepsilon$ respectively such that $\left|f_{\varepsilon}\left(z_{ \pm}\right)-( \pm 1)\right|<\varepsilon$ respectively. Since $R(\bar{U})=A(U)$ (see VIII.13 of [12]), we may use the Cauchy integral formula over $\partial U \backslash[-1 / 4,+1 / 4]$ to recapture values of $g$ on $U$,

$$
g(z)=\frac{1}{2 \pi i} \int_{\partial \Delta} \frac{g(\zeta)}{\zeta-z} d \zeta-\sum_{n=1}^{\infty} \frac{1}{2 \pi i} \int_{\partial \Delta_{n}} \frac{g(\zeta)}{\zeta-z} d \zeta, \quad z \in U
$$


Subtracting these expressions at $z_{+}$and $z_{-}$, we find that $g\left(z_{+}\right)-g\left(z_{-}\right)$is represented by a sum of integrals featuring $\left(z_{+}-z_{-}\right) g(\zeta) /\left(\zeta-z_{+}\right)\left(\zeta-z_{-}\right)$over the boundaries of the disks. Making the obvious estimates, we obtain a bound of the form

$$
\left|g\left(z_{+}\right)-g\left(z_{-}\right)\right| \leq c_{0}\|g\| \varepsilon .
$$

Thus if $\|g\| \leq 2$, then for $\varepsilon$ small,

$$
\left|\left(f_{\varepsilon}-g\right)\left(z_{+}\right)-\left(f_{\varepsilon}-g\right)\left(z_{-}\right)\right| \approx\left|f_{\varepsilon}\left(z_{+}\right)-f_{\varepsilon}\left(z_{-}\right)\right| \approx 2,
$$

and consequently $\left\|f_{\varepsilon}-g\right\| \geq 1 / 2$. On the other hand, if $\|g\| \geq 2$, then clearly $\left\|f_{\varepsilon}-g\right\| \geq 1 / 2$. Hence $\operatorname{dist}\left(f_{\varepsilon}, A(U)\right) \geq 1 / 2$ for $\varepsilon$ small.

Let $L_{0}^{\infty}(\sigma)$, vanishing $L^{\infty}$ of $\sigma$, denote the set of functions in $L^{\infty}(\sigma)$ that tend to 0 at $\partial U$. Any function in $L^{\infty}(\sigma)$ that is zero off some compact subset of $U$ belongs to $L_{0}^{\infty}(\sigma)$, and such functions are uniformly dense in $L_{0}^{\infty}(\sigma)$. Note that $L_{0}^{\infty}(\sigma)$ is a closed ideal of $L^{\infty}(\sigma)$. The analog of the preceding lemma for the linear span $H^{\infty}(U)+C(\bar{U})+L_{0}^{\infty}(\sigma)$ is as follows.

Lemma 2.3. The closed linear span $\left[H^{\infty}(U)+C(\bar{U})+L_{0}^{\infty}(\sigma)\right]$ coincides with $\left[H^{\infty}(U)+C(\bar{U})\right]+L_{0}^{\infty}(\sigma)$, and is a closed subalgebra of $L^{\infty}(\sigma)$. If $A(U)$ is pointwise boundedly dense in $H^{\infty}(U)$, then $H^{\infty}(U)+C(\bar{U})+L_{0}^{\infty}(\sigma)$ is already a closed subalgebra of $L^{\infty}(\sigma)$.

Proof. Since the sum of an algebra and an ideal is always an algebra, it suffices to show that $\left[H^{\infty}(U)+C(\bar{U})\right]+L_{0}^{\infty}(\sigma)$ is closed. Denote the functions in $C(\bar{U})$ that tend to 0 at $\partial U$ by $C_{0}(U)$. It is readily seen that for any function $f \in L^{\infty}(\sigma)$ that is continuous on $U$, the distances from $f$ to $L_{0}^{\infty}(\sigma)$ and to $C_{0}(U)$ both coincide with the limsup of $|f(z)|$ as $z$ tends to $\partial U$. In particular, the distances are equal. Since $C_{0}(U)=\left[H^{\infty}(U)+C(\bar{U})\right] \cap L_{0}^{\infty}(\sigma)$, Lemma 2.1 applies to $M=\left[H^{\infty}(U)+C(\bar{U})\right]$ and $N=L_{0}^{\infty}(\sigma)$, and the subspace sum is closed.

We will say that $z \in \partial U$ is a peak point for $H^{\infty}(U)$ if there is a function $f \in$ $H^{\infty}(U)$ such that $|f|<1$ on $U$ while $f(\zeta) \rightarrow 1$ as $\zeta \in U$ tends to $z$. If $z$ is not a peak point for $H^{\infty}(U)$, then a value $\varphi_{z}(f)$ can be assigned to $f$ at $z$ so that $f(\zeta)$ tends to $\varphi_{z}(f)$ as $\zeta$ tends to $z$ through a subset of $U$ with full area density at $z$. The functional $\varphi_{z}$ is a weak-star continuous multiplicative linear functional on $H^{\infty}(U)$, called the distinguished homomorphism at $z$. See [11] and [15] for background on distinguished homomorphisms.

\section{Multiplication Operators on $H^{\infty}(U)$}

Let $L_{s}^{\infty}(\sigma)$, slow $L^{\infty}$ of $\sigma$, denote the the set of functions $g \in L^{\infty}(\sigma)$ such that for every $\varepsilon>0$, there exists a subset $S$ of $U$ that is precompact in the norm of the dual space of $H^{\infty}(U)$ such that $|g|<\varepsilon$ off $S$. Thus a function $g \in L^{\infty}(\sigma)$ belongs to $L_{s}^{\infty}(\sigma)$ if and only if it has the following property: if $\zeta_{n} \in U$ converges to $z \in \partial U$, and if for some $c>0$ the essential limsup of $|g|$ at each $\zeta_{n}$ is $\geq c$, then $z$ is not a peak point for $H^{\infty}(U)$, and $\left\{\varphi_{\zeta_{n}}\right\}$ converges in norm to the distinguished homomorphism $\varphi_{z}$ at $z$. Any function in $L^{\infty}(\sigma)$ that is zero off some subset of $U$ that is precompact in the norm of the dual space of $H^{\infty}(U)$ belongs to $L_{s}^{\infty}(\sigma)$, and such functions are uniformly dense in $L_{s}^{\infty}(\sigma)$. Note that $L_{s}^{\infty}(\sigma)$ is a closed ideal of $L^{\infty}(\sigma)$. 
Theorem 3.1. For $g \in L^{\infty}(\sigma)$, define the multiplication operator $M_{g}$ from $H^{\infty}(U)$ to $L^{\infty}(\sigma)$ by $M_{g}(f)=g f$ for $f \in H^{\infty}(U)$. Then $M_{g}$ is compact if and only if $g \in L_{s}^{\infty}(\sigma)$. Further, if $M_{g}$ is not compact, then there is a subspace of $H^{\infty}(U)$ isomorphic to $\ell^{\infty}$ on which $M_{g}$ is an isomorphism.

Proof. Suppose that $g \in L_{s}^{\infty}(\sigma)$. Let $\left\{f_{n}\right\}$ be a sequence in the unit ball of $H^{\infty}(U)$ that converges uniformly to zero on compact subsets of $U$. We claim that $g f_{n}$ converges uniformly on $U$ to zero, from which it follows that $M_{g}$ is compact. Suppose on the contrary that there are $c>0$ and points $\zeta_{n} \in U$ such that the essential limsup of $\left|g f_{n}\right|$ at $\zeta_{n}$ is $\geq c$. Since $\left\|f_{n}\right\| \leq 1$ for all $n$, the essential limsup of $|g|$ at $\zeta_{n}$ is $\geq c$. Also note that $\|g\|\left|f_{n}\left(\zeta_{n}\right)\right| \geq c$, so $\left|f_{n}\left(\zeta_{n}\right)\right| \geq c /\|g\|$. But since the $f_{n}$ 's converge uniformly to zero on compact subsets of $U$, this means that the $\zeta_{n}$ 's do not remain within a compact subset of $U$, and so, by passing to a subsequence, we can assume that $\zeta_{n}$ converges to some $z \in \partial U$. Since $g \in L_{s}^{\infty}(\sigma)$, there is a distinguished homomorphism at $z$ and $\varphi_{\zeta_{n}} \rightarrow \varphi_{z}$ in norm, so that $\left|f_{n}\left(\zeta_{n}\right)-\varphi_{z}\left(f_{n}\right)\right| \rightarrow 0$. Since distinguished homomorphisms are weak-star continuous, $\varphi_{z}\left(f_{n}\right) \rightarrow 0$. Hence $f_{n}\left(\zeta_{n}\right) \rightarrow 0$, and this contradicts $\left|f_{n}\left(\zeta_{n}\right)\right| \geq c /\|g\|>0$.

Suppose on the other hand that $g \notin L_{s}^{\infty}(\sigma)$. Then there is a sequence $S$ in $U$ such that the essential limsup of $|g(z)|$ at each point of $S$ is $\geq c>0$, while the point evaluations at points of $S$ do not form a precompact subset of the dual space of $H^{\infty}(U)$. By Theorems 4.2 and 4.4 of [15], $S$ has an interpolating subsequence $\left\{z_{j}\right\}$, say with interpolating constant $M$. A theorem of Varopoulos (Theorem VII.2.2 of [16]) valid for arbitrary uniform algebras, together with a normal families argument to pass from a finite set to a sequence, provides us with a sequence of functions $f_{k} \in H^{\infty}(U)$ such that $f_{k}\left(z_{j}\right)=\delta_{j k}$ and $\sum\left|f_{k}\right| \leq M^{2}$ on $U$. The map $\alpha \mapsto F_{\alpha}=$ $\sum \alpha_{k} f_{k}$ of $\ell^{\infty}$ into $H^{\infty}(U)$ is then an embedding. Since $\left\|g F_{\alpha}\right\| \geq c\|\alpha\|, M_{g}$ is an isomorphism on the embedded copy of $\ell^{\infty}$.

\section{Hankel Operators with Symbols Vanishing on the Shilov Boundary}

Let $M$ be the maximal ideal space of $H^{\infty}(U)$. (We could as well use any compactification of $U$ to which the functions in $H^{\infty}(U)$ extend continuously and separate points.) The Shilov boundary of $H^{\infty}(U)$ is the smallest closed subset of $M$ on which all the functions in $H^{\infty}(U)$ attain their maximum modulus. We say that a function $g$ on $U$ tends to zero on the Shilov boundary if $g\left(z_{\alpha}\right) \rightarrow 0$ for any net $\left\{z_{\alpha}\right\}$ in $U$ that accumulates on the Shilov boundary in $M$. If $\Gamma$ is a free smooth boundary curve in $\partial U$, then $g$ tends to zero on the part of the Shilov boundary corresponding to $\Gamma$ if and only if $g$ has nontangential (or radial) boundary limit zero at almost all points of $\Gamma$. For background on the Shilov boundary of $H^{\infty}(U)$, see [11].

Theorem 4.1. Suppose $g \in L^{\infty}(\sigma)$ tends to zero on the Shilov boundary of $H^{\infty}(U)$. Then $S_{g}$ is compact if and only if $g \in L_{s}^{\infty}(\sigma)$. Further, if $S_{g}$ is not compact, then there is a subspace of $H^{\infty}(U)$ isomorphic to $\ell^{\infty}$ on which $S_{g}$ is an isomorphism.

Proof. For $g \in L_{s}^{\infty}(\sigma), M_{g}$ is compact by Theorem 3.1, so that $S_{g}$ is compact also. So suppose that $g \notin L_{s}^{\infty}(\sigma)$, while $g$ tends to zero on the Shilov boundary. By Theorem 3.1, there is a subspace $J$ of $H^{\infty}(U)$ isomorphic to $\ell^{\infty}$ on which $M_{g}$ is an isomorphism. Choose $c>0$ such that $\|g f\| \geq c\|f\|$ for all $f \in J$. Let $h \in H^{\infty}(U)$. Since $|g f-h|$ tends to $|h|$ on the Shilov boundary, $\|g f-h\| \geq\|h\|$. Also $\|g f-h\| \geq c\|f\|-\|h\|$. Adding these inequalities, we obtain $2\|g f-h\| \geq c\|f\|$. It follows that $2\left\|S_{g} f\right\| \geq c\|f\|$ for all $f \in J$, and $S_{g}$ is an isomorphism on $J$. 


\section{Construction of a Dual Basis}

It will be convenient to have other methods for introducing an $\ell^{\infty}$-basis into $H^{\infty}(U)$. Our basic technique is incorporated into the following lemma, which is a sharpened form of the dichotomy given in Theorems 4.2 and 4.4 of [15].

Lemma 5.1. Suppose $0 \in \partial U$, and let $\left\{\mu_{n}\right\}$ be a bounded sequence of measures on $U$ whose mass accumulates at 0 . Then:

(1) either $\left\{\mu_{n}\right\}$ forms a norm-precompact subset of the dual space of $H^{\infty}(U)$, in which case case either $\left\{\mu_{n}\right\}$ converges to 0 in the norm of the dual space of $H^{\infty}(U)$, or else 0 is a nonpeak point for $H^{\infty}(U)$ and $\left\{\mu_{n}\right\}$ accumulates in norm at multiples of the distinguished homomorphism $\varphi_{0}$;

(2) or else there are a constant $M>0$, a subsequence $\left\{\nu_{j}\right\}$ of $\left\{\mu_{n}\right\}$, and functions $f_{k} \in H^{\infty}(U)$ such that $\sum\left|f_{k}\right| \leq M$ on $U$ and $\int f_{k} d \nu_{j}=\delta_{j k}$ for all $j$ and $k$. In this case, given $\varepsilon_{k}>0, k \geq 1$, there are $r_{k}$ 's decreasing monotonically to 0 such that the $\nu_{j}$ 's and $f_{k}$ 's can be chosen as above so that additionally $f_{k}(\zeta)$ is analytic for $|\zeta|>r_{k}, f_{k}(\infty)=0, f_{k}(\zeta) \rightarrow 0$ as $\zeta \rightarrow 0$, and $\left|f_{k}(\zeta)\right|<\varepsilon_{k}$ for $|\zeta|<r_{k+1}$ and for $|\zeta|>r_{k}$.

Lemma 5.1 is related to the Rosenthal dichotomy: if $S$ is a bounded subset of a Banach space, then either every sequence in $S$ has a weakly Cauchy subsequence, or else $S$ contains a sequence that is an $\ell^{1}$-basis. Note that in the latter case, there is always a quotient space of the dual space that is isomorphic to $\ell^{\infty}$. Suppose now $S$ is the sequence $\left\{\mu_{n}\right\}$ of Lemma 5.1, regarded as a subset of the predual of $H^{\infty}(U)$. The first alternative of Lemma 5.1 corresponds to the first alternative of Rosenthal's dichotomy, with the stronger conclusion of norm compactness. The second alternative of the lemma asserts that not only is there a subset that forms an $\ell^{1}$-basis, but that the corresponding dual $\ell^{\infty}$ can be realized as a weak-star direct summand of the dual space $H^{\infty}(U)$.

The proof of Lemma 5.1 depends on a "sliding hump" argument, which appears frequently in analysis, and which is used in [15] and in [19]. Since the essential ideas of the proof are contained in Section 4 of [15], we give only an outline and omit some of the details.

The statements in the norm-precompact case follow directly from Section 4 of [15]. We assume then that the sequence is not precompact in the norm of the dual space of $H^{\infty}(U)$. We assume further that there is a distinguished homomorphism at 0 ; when 0 is a peak point the proof simplifies.

To fix notation, let $U_{\delta}=U \cup\{|z|>\delta\}$. If $\nu$ is a measure on $U$, let $\rho(\nu)$ denote the norm of $\nu$, regarded as a linear functional on the null-space of $\varphi_{0}$,

$$
\rho(\nu)=\sup \left\{\left|\int f d \nu\right|: f \in H^{\infty}(U),\|f\| \leq 1, \varphi_{0}(f)=0\right\} .
$$

We can assume here that $f$ is analytic at 0 and $f(0)=0$, since by [15] any $f$ in the null-space of $\varphi_{0}$ can be approximated uniformly on sets at a positive distance from 0 by functions of the same norm that are analytic at 0 . We can further assume that the original sequence satisfies $\rho\left(\mu_{n}\right) \geq c_{0}>0$, and we assume for convenience that $\left\|\mu_{n}\right\| \leq 1$. We use $C_{0}, C_{1}, \ldots$ to denote constants, which depend only on $c_{0}$.

Sublemma. Let $\delta>0$. For large $n$ there is $g_{n} \in H^{\infty}\left(U_{\delta}\right)$ such that $\left|g_{n}\right| \leq C_{1}$, $g_{n}(\infty)=0, g_{n}$ is analytic at $0, g_{n}(0)=0$, and $\int g_{n} d \mu_{n}=1$. 
Proof. Let $\phi=\phi_{\delta}$ be the usual bump function that is 1 near $z=0$ and 0 for $|z|>\delta$. Define $L_{\delta}(f)=T_{\phi}(f)-\phi f$. According to [15], for $\delta$ sufficiently small the norm of $L_{\delta}$ on the null-space of $\varphi_{0}$ tends to 0 with $\delta$. We choose $\delta$ small so that this norm is $<\varepsilon$. Let $f_{n} \in H^{\infty}(U)$ be such that $\left|f_{n}\right| \leq 1$ on $U, f_{n}$ is analytic at $0, f_{n}(0)=0$, and $\left|\int f_{n} d \mu_{n}\right| \geq c_{0} / 2$. Define $F_{n}=T_{\phi}\left(f_{n}\right)$. Then $F_{n} \in H^{\infty}\left(U_{\delta}\right),\left\|F_{n}\right\| \leq C_{0}, F_{n}(\infty)=0, F_{n}$ is analytic at 0 , and $\left|F_{n}(0)\right|<\varepsilon$. Further, $\int F_{n} d \mu_{n}=\int \phi f_{n} d \mu_{n}+\int L_{\delta}\left(f_{n}\right) d \mu_{n}$. Thus having fixed $\delta$ small, we obtain $\left|\int F_{n} d \mu_{n}\right|>c_{0} / 4$ for $n$ large, since the mass of $\mu_{n}$ accumulates at $z=0$ where $\phi=1$. Take any $h \in H^{\infty}\left(U_{\delta}\right)$ to be analytic at 0 , such that $h(0)=0$ and $h(\infty)=1$. Then $\int h d \mu_{n} \rightarrow 0$ as $n \rightarrow \infty$. If $h_{n}=F_{n}-F_{n}(0)+F_{n}(0) h$, then $g_{n}=h_{n} / \int h_{n} d \mu_{n}$ does the trick.

Sublemma. Let $\delta>0$. There is a subsequence $\left\{n_{j}\right\}$ such that for each nullsequence $s=\left\{s_{j}\right\} \in c_{0}$ with $\|s\| \leq 1$, there is $f \in H^{\infty}\left(U_{\delta}\right)$ such that $|f| \leq C_{2}$, $f(\infty)=0, f$ is continuous at $0, f(0)=0$, and $\int f d \mu_{n_{j}}=s_{j}, j \geq 1$.

Proof. Let $\varepsilon_{j}>0$ satisfy $\sum \varepsilon_{j}<1 / 2$. Proceeding by induction and applying the preceding sublemma, we obtain a sequence $\left\{\delta_{j}\right\}$ decreasing to 0 , a subsequence $\left\{n_{j}\right\}$, and functions $g_{j} \in H^{\infty}\left(U_{\delta}\right)$, such that $g_{j}(z)$ is analytic for $|z|>\delta_{j-1},\left|g_{j}\right| \leq C_{1}$, $g_{j}(\infty)=0, g_{j}(z)$ is analytic at $z=0, g_{j}(0)=0,\left|g_{j}(z)\right|<\varepsilon_{j}$ for $|z|<\delta_{j}$ and for $|z|>\delta_{j-1}, \int g_{j} d \mu_{n_{j}}=1$, and nearly all the mass of $\mu_{n_{j}}$ is carried by the annulus $\delta_{j}<|z|<\delta_{j-1}$. Here the estimate on $g_{j}(z)$ for $|z|>\delta_{j-1}$ is arranged at the induction step by requiring that $g_{j}(z)$ be analytic for $|z|>\varepsilon_{j} \delta_{j-1} / C_{1}$ and applying the Schwarz estimate. If now $s$ is a null sequence, then the series $\sum s_{j} g_{j}$ converges to a function $f_{1} \in H^{\infty}\left(U_{\delta}\right)$ such that $\left\|f_{1}\right\| \leq\left(C_{1}+1\right)\|s\|, f_{1}(\infty)=0, f_{1}$ is continuous at $0, f_{1}(0)=0$, and $\left|\int f_{1} d \mu_{n_{j}}-s_{j}\right| \leq\|s\| \sum \varepsilon_{j} \leq\|s\| / 2$. Next we apply the same procedure to the sequence $\left\{s_{j}-\int f_{1} d \mu_{n_{j}}\right\}$ and find $f_{2} \in H^{\infty}\left(U_{\delta}\right)$ such that $\left\|f_{2}\right\| \leq\left(C_{1}+1\right)\|s\| / 2, f_{2}(\infty)=0, f_{2}$ is continuous at $0, f_{2}(0)=0$, and $\left|\int f_{2} d \mu_{n_{j}}+\int f_{1} d \mu_{n_{j}}-s_{j}\right| \leq\|s\| / 4$. Iterating this procedure, we find that the function $f=\sum f_{k}$ has the desired properties, with $C_{2}=2\left(C_{1}+1\right)$.

Proof of Lemma 5.1. Suppose we have chosen $f_{1}, \ldots, f_{k-1}, r_{1}, \ldots, r_{k-1}$, and $\nu_{1}, \ldots, \nu_{k-1}$, so that upon relabeling we have $\mu_{j}=\nu_{j}$ for $1 \leq j \leq k-1$ and further $\int f_{j} d \mu_{n}=0$ for $n \geq k$ and $1 \leq j \leq k-1$. Choose $r_{k}>0$ small so that $\left|f_{k-1}(z)\right|<\varepsilon_{k-1}$ for $|z|<r_{k}$. Set $\delta_{k}=\varepsilon_{k} r_{k} / C_{1}$. For each infinite set of indices $J \subseteq\{k, k+1, \cdots\}$, consider the dimension of the space of $(k-1)$-vectors of the form $\left(\int h d \nu_{1}, \ldots, \int h d \nu_{k-1}\right)$, where $h \in H^{\infty}\left(U_{\delta}\right)$ is analytic for $|z|>\delta_{k}, h(z) \rightarrow 0$ as $z \rightarrow 0$, and $\int h d \mu_{n}=0$ for $n \in J$. We choose a subset $J$ such that this dimension is maximal, say $d$, and then we replace the $\mu_{n}$ 's for $n \geq k$ by the subsequence corresponding to $J$. There are then indices $1 \leq j_{1}<\cdots<j_{d} \leq k-1$ such that if $h \in H^{\infty}\left(U_{\delta}\right)$ is analytic for $|z|>\delta_{k}, h(z) \rightarrow 0$ as $z \rightarrow 0, \int h d \mu_{n}=0$ for an infinite set of $n$ 's that are $\geq k$, and $\int h d \mu_{j_{i}}=0$ for $1 \leq i \leq d$, then $\int h d \mu_{j}=0$ for all $j \geq 1$. Choose functions $h_{1}, \ldots, h_{d}$ with the above properties, that form a dual basis, so that $\int h_{m} d \mu_{j_{i}}=\delta_{m i}$ for $1 \leq m, i \leq d$. Pass to a subsequence of the $\mu_{n}$ 's for $n \geq k$ that has the properties of the preceding sublemma, applied with $\delta$ replaced by $\delta^{\prime}<\delta_{k}$. Let $\nu_{k}$ be the new $\mu_{k}$, and let $g_{k}$ be the function of the sublemma corresponding to the null-sequence $s=(1,0,0, \ldots)$, so that $\int g_{k} d \mu_{k}=1$ and $\int g_{k} d \mu_{n}=0$ for $n>k$. By choosing $\delta^{\prime}$ sufficiently small and invoking the Schwarz lemma, we can assume further that $\int g_{k} d \nu_{j_{i}}$ is very small for $1 \leq i \leq d$. 
Then

$$
f_{k}=g_{k}-\sum_{i=1}^{d}\left(\int g_{k} d \mu_{j_{i}}\right) h_{i}
$$

has the properties required for the induction step, and Lemma 5.1 is proved.

Lemma 5.2. Let $g \in L^{\infty}(\sigma)$, and let $\left\{\mu_{n}\right\}$ be a bounded sequence of measures on $U$ whose mass accumulates at $z \in \partial U$, such that $\mu_{n} \perp H^{\infty}(U)$. Suppose there are a constant $M>0$ and functions $f_{k} \in H^{\infty}(U)$ such that $\sum\left|f_{k}\right| \leq M$ on $U$ and $\int g f_{k} d \mu_{j}=\delta_{j k}$ for all $j$ and $k$. Then the map $\alpha \mapsto F_{\alpha}=\sum \alpha_{k} f_{k}$ is an embedding of $\ell^{\infty}$ into $H^{\infty}(U)$, and $S_{g}$ is an isomorphism on the embedded copy of $\ell^{\infty}$.

Proof. The map is evidently an embedding onto a weak-star closed subspace isomorphic to $\ell^{\infty}$, on which $S_{g}$ is bounded. The estimate $\left|\alpha_{n}\right|=\left|\int g F_{\alpha} d \mu_{n}\right| \leq$ $\left\|g F_{\alpha}+H^{\infty}(U)\right\|\left\|\mu_{n}\right\|$ shows that $\|\alpha\| \leq C\left\|S_{g}\left(F_{\alpha}\right)\right\|$. Thus $S_{g}$ is bounded below, hence an isomorphism.

\section{The Algebra of Symbols of Compact Hankel Operators}

Denote by $B^{\infty}(U)$ the set of all $g \in L^{\infty}(\sigma)$ such that the Hankel operator $S_{g}$ is compact. Thus $g \in B^{\infty}(U)$ if and only if $g f_{n}+H^{\infty}(U)$ converges to zero in the norm of $L^{\infty}(\sigma) / H^{\infty}(U)$ whenever $\left\{f_{n}\right\}$ is a bounded sequence in $H^{\infty}(U)$ that converges pointwise to zero on $U$ (= uniformly to zero on compact subsets of $U$ ). Evidently $B^{\infty}(U)$ is a closed subalgebra of $L^{\infty}(\sigma)$.

A closed subalgebra of $L^{\infty}(\sigma)$ is said to be $T$-invariant if for any smooth function $\phi$ with compact support in the complex plane, the algebra is invariant under the operator $T_{\phi}$ defined in the proof of Lemma 2.2. The operators $T_{\phi}$ are the localization operators used in rational approximation theory, which solve the delta-bar problem $\bar{\partial} T_{\phi} g=\phi \bar{\partial} g$. For a discussion of these operators and their applications in rational approximation theory, see [9] or [14].

Lemma 6.1. The algebra $B^{\infty}(U)$ is T-invariant.

Proof. Let $g \in B^{\infty}(U)$. Suppose $\left\{f_{n}\right\}$ is a bounded sequence in $H^{\infty}(U)$ that converges pointwise to zero on $U$. We must show that $\left\|T_{\phi}(g) f_{n}+H^{\infty}(U)\right\| \rightarrow 0$. Choose $h_{n} \in L^{1}(\sigma)$ such that $h_{n} \perp H^{\infty}(U),\left\|h_{n}\right\|_{1} \leq 1$, and

$$
\left\|T_{\phi}(g) f_{n}+H^{\infty}(U)\right\|=\int h_{n} T_{\phi}(g) f_{n} d \sigma .
$$

Using the definition of $T_{\phi}$, we can express this integral in the form

$$
\int h_{n} T_{\phi}\left(g f_{n}\right) d \sigma+\frac{1}{\pi} \iiint h_{n}(\zeta) g(z) \frac{f_{n}(\zeta)-f_{n}(z)}{z-\zeta} \frac{\partial \phi}{\partial \bar{z}} d x d y d \sigma(\zeta) .
$$

Interchanging the orders of integration and using $h_{n} \perp H^{\infty}(U)$, we see that the last integral is zero. The first integral is estimated by $\left\|T_{\phi}\right\|\left\|g f_{n}+H^{\infty}(U)\right\|$, which tends to zero since $g \in B^{\infty}(U)$.

Lemma 6.2. If $g \in C(\bar{U})$, then $S_{g}$ is compact. Thus

$$
\left[H^{\infty}(U)+C(\bar{U})+L_{s}^{\infty}(\sigma)\right] \subseteq B^{\infty}(U) .
$$

Proof. If $g \in L_{s}^{\infty}(\sigma)$, then $M_{g}$ is compact by Theorem 3.1, so that $S_{g}$ is compact. Also $S_{g}=0$ for $g \in H^{\infty}(U)$. Thus the second assertion of the lemma follows from the first. To prove the first assertion, we use the fact (see [9]) that the linear span 
of $A(U)$ and $\left\{1 /\left(\zeta-z_{0}\right): z_{0} \in U\right\}$ is dense in $C(\partial U)$. Thus it suffices to show that $S_{g}$ is compact for any $g \in L^{\infty}(\sigma)$ that coincides in some neighborhood of $\partial U$ with $1 /\left(\zeta-z_{0}\right)$. So fix $z_{0} \in U$ and such a $g$, and let $\left\{f_{n}\right\}$ be a bounded sequence in $H^{\infty}(U)$ that converges pointwise to zero on $U$. Then the functions

$$
h_{n}(\zeta)=g(\zeta)\left[f_{n}(\zeta)-f_{n}\left(z_{0}\right)\right]-\left[f_{n}(\zeta)-f_{n}\left(z_{0}\right)\right] /\left(\zeta-z_{0}\right)
$$

vanish off a fixed compact subset of $U$, and $h_{n} \rightarrow 0$ uniformly on $U$. Since $g f_{n} \equiv$ $f_{n}\left(z_{0}\right) g+h_{n}$ modulo $H^{\infty}(U)$, we obtain $\left\|g f_{n}+H^{\infty}(U)\right\| \rightarrow 0$. Hence $S_{g}$ is compact.

Now we aim towards finding a converse to Lemma 6.2. For this it will be convenient to work on the spectrum (maximal ideal space) of $L^{\infty}(\sigma)$, which we denote by $\Sigma$. Thus $L^{\infty}(\sigma) \cong C(\Sigma)$. The fiber of $\Sigma$ over a point $z \in \bar{U}$ is denoted by $\Sigma_{z}$ and consists of all homomorphisms $\varphi \in \Sigma$ such that $\varphi(\zeta)=z$, where $\zeta$ is the identity function on $\mathbb{C}$. We regard $\left[H^{\infty}(U)+C(\bar{U})+L_{0}^{\infty}(\sigma)\right]$ as a closed subalgebra of $C(\Sigma)$. Since $C(\bar{U})+L_{0}^{\infty}(\sigma)$ is a self-adjoint subalgebra, the Shilov decomposition theorem, or Bishop's antisymmetric decomposition theorem, shows that the algebra consists precisely of all $f \in C(\Sigma)$ such that $f\left|\Sigma_{z} \in H^{\infty}(U)\right| \Sigma_{z}$ for all $z \in \partial U$.

The Shilov decomposition theorem also shows that $B^{\infty}(U)$ consists precisely of all $g \in C(\Sigma)$ such that $g\left|\Sigma_{z} \in B^{\infty}(U)\right| \Sigma_{z}$ for all $z \in \partial U$. Thus our task is to determine when $B^{\infty}(U)\left|\Sigma_{z}=H^{\infty}(U)\right| \Sigma_{z}$.

The fiber algebra $H^{\infty}(U) \mid \Sigma_{z}$ depends only on the local character of $U$ at $z$ (see [13]). The following lemma shows that the same is true of the fiber algebra $B^{\infty}(U) \mid \Sigma_{z}$.

Lemma 6.3. Fix $z \in \partial U$. Let $\delta>0$ and set $V=U \cap \Delta(z, \delta)$. Then there is a natural identification of the fibers of $\Sigma$ over $z$ associated with $U$ and $V$, and under this identification the algebras $B^{\infty}(U) \mid \Sigma_{z}$ and $B^{\infty}(V) \mid \Sigma_{z}$ coincide.

Proof. The proof given in [13] for $H^{\infty}(U)$ depends only on the $T$-invariance of $H^{\infty}(U)$, and hence in view of Lemma 6.1 it carries over to $B^{\infty}(U)$. We omit the details.

Our basic dichotomy now takes the following form.

Theorem 6.4. Let $g \in L^{\infty}(\sigma)$ and $z \in \partial U$ be such that $g\left|\Sigma_{z} \notin H^{\infty}(U)\right| \Sigma_{z}$. Then either $z$ is a nonpeak point for $H^{\infty}(U)$ and there is a bounded sequence of functions $h_{n} \in L^{1}(\sigma)$ such that $h_{n}=0$ outside the disk $\Delta(z, 1 / n), h_{n} \perp H^{\infty}(U)$, and $h_{n} g d \sigma \rightarrow \varphi_{z}$ in the norm of the dual of $H^{\infty}(U)$; or else there are a constant $M>0$, a bounded sequence of functions $\left\{h_{n}\right\}$ in $L^{1}(\sigma)$, and functions $f_{k} \in H^{\infty}(U)$ such that $\sum\left|f_{k}\right| \leq M<\infty, h_{n}=0$ outside the disk $\Delta(z, 1 / n), h_{n} \perp H^{\infty}(U)$, and $\int f_{k} g h_{n} d \sigma=\delta_{k n}$. In the latter case, the map $\alpha \mapsto F_{\alpha}=\sum \alpha_{k} f_{k}$ is an embedding of $\ell^{\infty}$ into $H^{\infty}(U)$, and $S_{g}$ is an isomorphism on the embedded copy of $\ell^{\infty}$.

Proof. Let $\nu$ be a measure on $\Sigma_{z}$ such that $\nu \perp H^{\infty}(U)$ and $\int g d \nu=1$. Since the unit ball of the predual of $H^{\infty}(U)$ is weak-star dense in the unit ball of the dual, there is a function $h \in L^{1}(\sigma)$ such that $\|h\|_{1} \leq\|\nu\|+\varepsilon, h \perp H^{\infty}(U)$, and $\int g h d \sigma=1$. By Lemma 6.3 we can apply this to $U \cap \Delta(z, 1 / n)$ for each $n \geq 1$. Denote by $h_{n}$ the corresponding function, extended to be zero off $\Delta(z, 1 / n)$. Then $\left\{h_{n}\right\}$ is a bounded sequence in $L^{1}(\sigma), h_{n} \perp H^{\infty}(U)$, and $\int h_{n} g d \sigma=1$. We apply Lemma 5.1 to the measures $\mu_{n}=h_{n} g d \sigma$. If the $\mu_{n}$ 's are norm precompact, then the condition on the integrals and Lemma 5.1 show that the only norm limit point can 
be a distinguished homomorphism, so that by Lemma 5.1 the first alternative of the theorem obtains. If the $\mu_{n}$ 's are not norm precompact, then the second alternative of Lemma 5.1 yields upon passing to a subsequence the second alternative of the theorem. The final statement is Lemma 5.2.

Theorem 6.5. Let $z \in \partial U$. Then $B^{\infty}(U)\left|\Sigma_{z}=H^{\infty}(U)\right| \Sigma_{z}$ if and only if $z$ is a peak point for $H^{\infty}(U)$.

Proof. Suppose the two fiber algebras are distinct. By Lemma 6.2 and the discussion following it, there is $g \in B^{\infty}(U)$ such that $g\left|\Sigma_{z} \notin H^{\infty}(U)\right| \Sigma_{z}$. Since $S_{g}$ is compact, the second alternative of Theorem 6.4 cannot occur, and consequently $z$ is a nonpeak point. Conversely, suppose $z$ is a nonpeak point. By [15] we can find small disks $D_{n}$ in $U$ that accumulate at $z$, such that the point evaluations at points of the $D_{n}$ 's converge to the distinguished homomorphisn $\varphi_{z}$ in the norm of the dual of $H^{\infty}(U)$. Define $g$ to be 1 on the $D_{n}$ 's and 0 elsewhere. Then $g \in L_{s}^{\infty}(\sigma) \subseteq B^{\infty}(U)$, and $g$ assumes both the values 0 and 1 on $\Sigma_{z}$. By Iversen's theorem (see [11]), $g=0$ on the Shilov boundary of the fiber algebra $H^{\infty}(U) \mid \Sigma_{z}$. Hence $g\left|\Sigma_{z} \notin H^{\infty}(U)\right| \Sigma_{z}$.

From the "if" statement of Theorem 6.5 we deduce easily the following converse to Lemma 6.2.

Theorem 6.6. Suppose every point of $\partial U$ is a peak point for $H^{\infty}(U)$. Let $g \in$ $L^{\infty}(\sigma)$. Then $S_{g}$ is compact if and only if $g \in\left[H^{\infty}(U)+C(\bar{U})+L_{0}^{\infty}(\sigma)\right]$. Further, if $S_{g}$ is not compact, then there is a subspace of $H^{\infty}(U)$ isomorphic to $\ell^{\infty}$ on which $S_{g}$ is an isomorphism.

Proof. In this case there are no distinguished homomorphisms, and $L_{s}^{\infty}(\sigma)=$ $L_{0}^{\infty}(\sigma)$. By Theorem $6.5, B^{\infty}(U)\left|\Sigma_{z}=H^{\infty}(U)\right| \Sigma_{z}$ for all $z \in \partial U$. The remarks preceding Lemma 6.3 then show that $B^{\infty}(U)=\left[H^{\infty}(U)+C(\bar{U})+L_{0}^{\infty}(\sigma)\right]$. For the second statement, note that if $S_{g}$ is not compact, there is $z \in \partial U$ such that $g\left|\Sigma_{z} \notin H^{\infty}(U)\right| \Sigma_{z}$. The second alternative of Theorem 6.4 then gives the embedding.

\section{Behrens Roadrunner Domains}

In this section we fix $D$ to be a Behrens roadrunner domain, that is, $D$ is a subdomain of the punctured open unit disk $\Delta \backslash\{0\}$ obtained by excising a sequence of disjoint closed disks $\bar{\Delta}_{n}$, where the $\Delta_{n}$ 's have centers $c_{n}$ and radii $r_{n}$ tending to 0 , and further there are $R_{0}<1$ and $R_{n}>r_{n}, n \geq 1$, such that $\sum r_{n} / R_{n}<\infty$ and the annular collars $\left\{R_{0}<|z|<1\right\}$ and $\left\{r_{n}<\left|z-c_{n}\right|<R_{n}\right\}, n \geq 1$, are disjoint. Thus the boundary of $D$ consists of $\{0\}$, the unit circle $\{|z|=1\}$, and the circles $\left\{\left|z-c_{n}\right|=r_{n}\right\}$ for $n \geq 1$. The summability condition guarantees that $\sum r_{n} /\left|c_{n}\right|<\infty$. Hence $(1 / z) d z$ is a finite measure on $\partial D$, which then represents a distinguished homomorphism $\varphi_{0}$ at 0 ,

$$
\varphi_{0}(f)=\frac{1}{2 \pi i} \int_{\partial D} \frac{f(z)}{z} d z, \quad f \in H^{\infty}(D) .
$$

We define an ample collar for $\partial D$ to be an open subset of $D$ that is a union of disjoint annuli $\left\{s_{0}<|z|<1\right\}$ and $\left\{r_{n}<\left|z-c_{n}\right|<s_{n}\right\}, n \geq 1$, where $R_{0}<s_{0}<1$, $r_{n}<s_{n}<R_{n}$ for $n \geq 1$, and $s_{n} / r_{n} \rightarrow \infty$. The estimates in [2] show that a sequence of points $z_{n} \in D$ with $\left|z_{n}\right| \rightarrow 0$ converges to the distinguished homomorphism $\varphi_{0}$ 
in norm if and only if there is an ample collar for $\partial D$ that contains no point of the sequence. Thus any function in $L^{\infty}(\sigma)$ that is zero on some ample collar belongs to $L_{s}^{\infty}(\sigma)$, and such functions are dense in $L_{s}^{\infty}(\sigma)$.

Let $V H^{\infty}(D)$, varying $H^{\infty}$ of $D$, denote the set of functions $g \in L^{\infty}(\sigma)$ for which there is an ample collar $\left\{A_{n}\right\}$ for $\partial D$ such that $g=0$ off the union of the $A_{n}$ 's, while $g$ is analytic on each $A_{n}$. Clearly $V H^{\infty}(D)+L_{s}^{\infty}(\sigma)$ is a subalgebra of $L^{\infty}(\sigma)$, though $V H^{\infty}(D)$ itself is not even a linear subspace of $L^{\infty}(\sigma)$.

Theorem 7.1. Let $D$ be a Behrens roadrunner domain, and let $g \in L^{\infty}(\sigma)$. Then $S_{g}$ is compact if and only if $g \in V H^{\infty}(D)+C(\bar{D})+L_{s}^{\infty}(\sigma)$. Further, if $S_{g}$ is not compact, then there is a subspace of $H^{\infty}(D)$ isomorphic to $\ell^{\infty}$ on which $S_{g}$ is an isomorphism.

Proof. For the first part of the proof, we fix $g \in L^{\infty}(\sigma)$, and we consider three cases.

Suppose first that there is some $z \in \partial D, z \neq 0$, such that $g\left|\Sigma_{z} \notin H^{\infty}(D)\right| \Sigma_{z}$. Since $z$ is a peak point, the second alternative of Theorem 6.4 applies, and there is a subspace of $H^{\infty}(D)$ isomorphic to $\ell^{\infty}$ on which $S_{g}$ is an isomorphism. In particular, $S_{g}$ is not compact.

Suppose next that there are $c>0$ and $\alpha>1$ such that the limsup of the distances from $g$ to $H^{\infty}\left(E_{n}\right)$ is $\geq c$, where $E_{n}$ denotes the annulus $\left\{r_{n}<\left|z-c_{n}\right|<\alpha r_{n}\right\}$. Note that these annuli are disjoint subsets of $D$ for $n$ large, and we consider only such $n$. By duality there are functions $h_{n} \in L^{1}\left(\sigma \mid E_{n}\right)$ such that $h_{n} d \sigma \perp H^{\infty}\left(E_{n}\right)$, $\int\left|h_{n}\right| d \sigma=1$, and the limsup of $\left|\int g h_{n} d \sigma\right|$ is $\geq c$, the integrals being carried over $E_{n}$. Consider the functions $L_{k}(z)=r_{k} /\left(z-c_{k}\right)$, which are invertible in $H^{\infty}(D)$ and satisfy $\left\|L_{k}\right\|=1$ and $\left|L_{k}(z)\right| \geq 1 / \alpha$ for $z \in E_{k}$. We apply Lemma 5.1 to the measures $\mu_{n}=L_{n}^{-1} g h_{n} d \sigma$ for those $n$ for which $\left|\int g h_{n} d \sigma\right|>c / 2$. Assume the $\mu_{n}$ 's form a norm-precompact subset of the dual of $H^{\infty}(D)$. By Lemma 5.1, there is then a subsequence that converges in the norm of the dual of $H^{\infty}(D)$ to a constant multiple $\beta \varphi_{0}$ of the distinguished homomorphism $\varphi_{0}$. However, this leads to $\left|\int g h_{n} d \sigma\right|=\left|\int L_{n} d \mu_{n}\right| \leq|| \mu_{n}-\beta \varphi_{0}||+\left|\beta L_{n}(0)\right| \rightarrow 0$, which is a contradiction. We conclude that the second alternative of Lemma 5.1 holds, and then we apply Lemma 5.2 to obtain a copy of $\ell^{\infty}$ in $H^{\infty}(D)$ on which $S_{g}$ is an isomorphism. We remark in passing that the embedding of $\ell^{\infty}$ can be constructed directly to have the form $\alpha \mapsto \sum \alpha_{n} L_{n}$, where the indices are restricted to an appropriate set of positive integers.

The only remaining possibility is that $g\left|\Sigma_{z} \in H^{\infty}(D)\right| \Sigma_{z}$ for all $z \in \partial D, z \neq 0$, and there is a sequence $\alpha_{n} \rightarrow \infty$ such that the annuli $E_{n}=\left\{r_{n}<\left|z-c_{n}\right|<\alpha_{n} r_{n}\right\}$ are disjoint and the distance from $g$ to $H^{\infty}\left(E_{n}\right)$ tends to zero as $n \rightarrow \infty$. We claim in this case that $g \in V H^{\infty}(D)+C(\bar{D})+L_{s}^{\infty}(\sigma)$.

Note that the $E_{n}$ 's form an ample collar. By subtracting from $g$ a function in $V H^{\infty}(D)$, we can assume that supremum of $|g|$ over $E_{n}$ tends to 0 as $n \rightarrow \infty$. By replacing $g$ by its restriction to a slightly smaller ample collar, which amounts to subtracting a function in $L_{s}^{\infty}(\sigma)$, we can assume that $g=0$ outside the collar and in a neighborhood of the boundary curves of the $E_{n}$ 's in $D$. Then for each $n$ we have $g \mid E_{n} \in B^{\infty}\left(E_{n}\right)$, which by Theorem 6.6 and Lemma 2.3 coincides with $H^{\infty}\left(E_{n}\right)+C\left(\overline{E_{n}}\right)+L_{0}^{\infty}\left(E_{n}\right)$. Thus on $E_{n}$ we can express $g=h_{n}+\varphi_{n}+\psi_{n}$, where $h_{n} \in H^{\infty}\left(E_{n}\right), \varphi_{n} \in C\left(\overline{E_{n}}\right)$, and $\psi_{n} \in L_{0}^{\infty}\left(E_{n}\right)$. Further, the summands can be chosen so that their norms on the $E_{n}$ 's are bounded by the norms of $g$ there and hence tend to zero as $n \rightarrow \infty$. In fact, we replace $\varphi_{n}$ by the Poisson integral of its 
boundary values and make the corresponding alteration in $\psi_{n}$, and then $h_{n}+\varphi_{n}$ is the Poisson integral of its nontangential boundary values, hence bounded by the norm of $g$, so $\psi_{n}$ is bounded by twice the norm of $g$. Since the distance from $\varphi_{n}$ to $H^{\infty}\left(E_{n}\right)$ is equal to its distance to the analytic functions with continuous boundary values, we can further modify $\varphi_{n}$ and $h_{n}$ so that $\varphi_{n}$ is bounded by $1+\varepsilon$ times the norm of $g$, and then $h_{n}$ is bounded by $2+\varepsilon$ times the norm of $g$ on $E_{n}$. Since now the norms over the $E_{n}$ 's tend to zero, the $\varphi_{n}$ 's can be extended to $\varphi \in C(\bar{D})$, the $\psi_{n}$ 's to $\psi \in L_{s}^{\infty}(\sigma)$, and $h_{n}$ to $h \in V H^{\infty}(D)$, so that $g=h+\varphi+\psi$ on $\bigcup E_{n}$. It follows that $g \in V H^{\infty}(D)+C(\bar{D})+L_{s}^{\infty}(\sigma)$, as asserted.

Now we work the condition from the other end and show that $S_{g}$ is compact whenever $g \in V H^{\infty}(D)+C(\bar{D})+L_{s}^{\infty}(\sigma)$. Since $S_{g}$ is compact for all $g \in C(\bar{D})+$ $L_{s}^{\infty}(\sigma)$, it suffices to establish compactness when $g \in V H^{\infty}(D)$. So fix such a $g$, and let $\left\{A_{m}\right\}$ be an ample collar for $\partial D$ such that $g=0$ off $\bigcup A_{m}$, and $g$ is an analytic function $g_{m}$ on $A_{m}$. We may shrink the $A_{m}$ 's to a smaller ample collar and crop $g$ whenever it is convenient, since the cropped $g$ differs from the original $g$ by a function in $L_{s}^{\infty}(\sigma)$.

Suppose that $g_{m}=0$ for all but finitely many $m$ 's. Then we can find $G$ continuous on $D$ such that $G=g_{m}$ near the $m$ th boundary circle, and $G=0$ near $z=0$. Then $G \in H^{\infty}+C$ at each boundary point. Since $H^{\infty}+C$ is local, $G \in H^{\infty}(D)+C(\bar{D})$, and $S_{G}$ is compact. Hence $S_{g}$ is compact.

For the remaining steps we will use the Laurent decomposition for annuli, decomposing an analytic function $f$ as a sum of a function analytic inside the outer circle plus a function analytic outside the inner circle and at $\infty$. There is a normalization, either to vanish at $\infty$ or to vanish at the center of the circles. The summands are both bounded by $C\|f\|_{\infty}$, and the constant $C$ is uniformly bounded provided the module of the annulus remains bounded away from 1 . Since we deal with annuli whose modules are bounded away from 1, we will use the same $C$ for all our Laurent decompositions.

Resuming the proof, we assume next that each $g_{m}$ extends from $A_{m}$ to be analytic for $\left|z-c_{m}\right|>r_{m}$ and at $\infty$; denote the extension also by $g_{m}$. Assume also for convenience that $\|g\| \leq 1$. Let $\varepsilon>0$. Choose $N \geq 1$ such that $R_{n}>2 r_{n}$ for $n \geq N$ and

$$
C \sum_{n=N}^{\infty} r_{n} / R_{n}<\varepsilon
$$

Choose $N$ also so that if $f \in H^{\infty}(D)$ satisfies $\|f\| \leq 1$ and $\varphi_{0}(f)=0$, then $|f(z)|<\varepsilon$ for $R_{n} / 2 \leq\left|z-c_{n}\right| \leq R_{n}, n \geq N$. Let $F_{n}$ be the outer component of the Laurent decomposition of $f$ with respect to the annulus $A_{n}$, normalized so that $F_{n}(\infty)=0$. The Cauchy integral formula

$$
F_{n}(z)=\frac{1}{2 \pi i} \int_{\left|z-c_{n}\right|=R_{n} / 2} \frac{f(\zeta)}{\zeta-z} d \zeta, \quad\left|z-c_{n}\right|>R_{n} / 2
$$

and the obvious estimate yield $\left|F_{n}(z)\right| \leq \varepsilon$ for $\left|z-c_{n}\right|=R_{n}$. Thus $\left|f(z)-F_{n}(z)\right| \leq$ $2 \varepsilon$ for $\left|z-c_{n}\right|=R_{n}$, hence for $\left|z-c_{n}\right| \leq R_{n}$, by the maximum modulus principle. Define $h(z)=g(z)$ for $z \in A_{n}, n \geq N$, and set $h(z)=0$ elsewhere. Since $g-h$ vanishes off all but finitely many annular collars, $S_{g-h}$ is compact. Set $F(z)=$ $\sum_{k=N}^{\infty} g_{k} F_{k}$. If $z \in A_{n}$ for some fixed $n \geq N$, we estimate

$$
|h(z) f(z)-F(z)| \leq\left|f(z)-F_{n}(z)\right|+\sum_{k \geq N, k \neq n}\left|F_{k}(z)\right| .
$$


The first term on the right is estimated by $2 \varepsilon$. The Schwarz inequality gives $\left|F_{k}(z)\right| \leq C r_{k} / R_{k}$ for $z \notin A_{k}$, so that our condition on $N$ shows that the sum on the right is bounded by $\varepsilon$. Thus $|h(z) f(z)-F(z)| \leq 3 \varepsilon$ for $z \in A_{n}, n \geq N$. If $z \notin \bigcup_{n \geq N} A_{n}$, then $h=0$ and $|h(z) f(z)-F(z)|=|F(z)| \leq \sum_{k \geq N}\left|F_{k}(z)\right| \leq \varepsilon$. Thus $\|h f-F\| \leq 3 \varepsilon$. Since $F \in H^{\infty}(D)$, we obtain $\left\|S_{h} f\right\| \leq 3 \varepsilon$. Since $S_{g-h}$ is compact, the distance from $S_{g}$ to the compacts, regarded as operators on the kernel of $\varphi_{0}$, is $\leq 3 \varepsilon$. It follows that $S_{g}$ is compact.

Now we turn to an arbitrary $g \in V H^{\infty}(D)$. For convenience we subtract the compact operator corresponding to $g_{0}$ and assume that $g_{0}=0$. For $m \geq 1$, let $G_{m}$ be the inside component of the Laurent decomposition of $g_{m}$ with respect to the annulus $A_{m}$, normalized so that $G_{m}\left(c_{m}\right)=0$. Then $G_{m}(z)$ is analytic for $\left|z-c_{m}\right|<$ $R_{m}$, and $\left|G_{m}\right| \leq C|| g||$. By the Schwarz inequality, $\left|G_{m}(z)\right| \leq C|| g||\left|z-c_{m}\right| / R_{m}$. Choose $r_{m}<s_{m}<R_{m}$ such that $s_{m} / r_{m} \rightarrow \infty$ while $s_{m} / R_{m} \rightarrow 0$. Define an analytic function $G$ on the union of the annuli $\left\{r_{m}<\left|z-c_{m}\right|<s_{m}\right\}$ by setting $G(z)=G_{m}(z)$ for $r_{m}<\left|z-c_{m}\right|<s_{m}$. Then $G(z) \rightarrow 0$ as $z \rightarrow 0$. By decreasing the $s_{m}$ 's slightly, we can assume that $G_{m}$ is continuous at the circle $\left|z-c_{m}\right|=s_{m}$, and then we can extend $G$ to be continuous on $\bar{D}$. Consequently $S_{G}$ is compact. In view of the preceding paragraph, $S_{g-G}$ is compact. Hence $S_{g}$ is compact.

\section{Hankel Operators Defined with Respect to Harmonic Measure}

In this section we indicate how to obtain results in the context of harmonic measure on the boundary rather than area measure on the domain.

One strategy for passing between the interior and the boundary is through asymptotic multiplicative properties of the Poisson kernel $P$. For example, if the map $f \rightarrow P(g) P(f)-P(g f)+H^{\infty}$ were compact for all $g \in L^{\infty}(\partial D)$, then one could transfer results from the interior to the boundary. Unfortunately this map is not always compact. In fact, in the case of the unit disk this map is compact if and only if $g \in H^{\infty}+C$. (See [18].) It does turn out, however, that results for boundary measures corresponding to the results obtained above for interior measures are valid, and they can be established by following the same method of proof as used above for the area measure. We sketch the procedure.

Let $\mu$ denote harmonic measure on the boundary of $U$, and let $H^{\infty}(\mu)$ denote the subalgebra of $L^{\infty}(\mu)$ of functions whose Poisson integrals are analytic on $U$. Then $H^{\infty}(\mu)$ is isometrically isomorphic and weak-star homeomorphic to a weakstar closed subalgebra of $H^{\infty}(U)$. In the case that the boundary of $U$ consists of a finite number of free simple closed curves, or in the case in which $U$ is a Behrens roadrunner domain, every function in $H^{\infty}(U)$ is the Poisson integral of a function in $L^{\infty}(\mu)$, so that $H^{\infty}(\mu)$ is isometrically isomorphic and weak-star homeomorphic to $H^{\infty}(U)$ via the Poisson integral.

As before, we define the Hankel operator $S_{g}$ from $H^{\infty}(\mu)$ to $L^{\infty}(\mu) / H^{\infty}(\mu)$ by $S_{g}(f)=g f+H^{\infty}(\mu)$. The set of $g \in L^{\infty}(\mu)$ such that $S_{g}$ is compact forms a closed subalgebra of $L^{\infty}(\mu)$, which is $T$-invariant and hence local. The dichotomy given by Lemma 5.1 remains valid for any bounded sequence of measures on $\partial U$ that is absolutely continuous with respect to $\mu$. The analog of Theorem 6.6 assumes the following form.

Theorem 8.1. Suppose that every point of $\partial U$ is a peak point for $H^{\infty}(\mu)$. Let $g \in L^{\infty}(\mu)$. Then $S_{g}$ is compact if and only if $g \in\left[H^{\infty}(\mu)+C(\partial U)\right]$. Further, if 
$S_{g}$ is not compact, then there is a subspace of $H^{\infty}(\mu)$ isomorphic to $\ell^{\infty}$ on which $S_{g}$ is an isomorphism.

Theorem 8.1 applies to the unit disk, and more generally it applies whenever the boundary of $U$ consists of a finite number of free simple closed curves. We focus on the case in which $U$ is a Behrens roadrunner domain, as defined in Section 7.

With the notation of Section 7 , we define $V H^{\infty}(\mu)$, varying $H^{\infty}$ of harmonic measure, to be the set of functions $g \in L^{\infty}(\mu)$ such that $g \mid \partial \Delta$ is the boundary values of a bounded analytic function on the open unit disk, and for each $n \geq 1$, the restriction $g \mid \partial \Delta_{n}$ is the restriction to $\partial \Delta_{n}$ of a bounded analytic function in the exterior domain $\mathbb{C}^{*} \backslash \bar{\Delta}_{n}$. This does not coincide exactly with the definition of $V H^{\infty}(D)$ given earlier, but it does coincide modulo $C(\partial D)$. Evidently $V H^{\infty}(\mu)$ is a closed subalgebra of $L^{\infty}(\mu)$. It is not hard to see that $V H^{\infty}(\mu)+C(\partial D)$ coincides with the space of nontangential boundary-value functions of functions in $V H^{\infty}(D)+C(\bar{D})+L_{s}^{\infty}(\sigma)$.

Theorem 8.2. Let $D$ be a Behrens roadrunner domain, let $\mu$ be harmonic measure on the boundary of $D$, and let $g \in L^{\infty}(\mu)$. Then $S_{g}$ is compact if and only if $g \in V H^{\infty}(\mu)+C(\partial D)$. Further, if $S_{g}$ is not compact, then there is a subspace of $H^{\infty}(\mu)$ isomorphic to $\ell^{\infty}$ on which $S_{g}$ is an isomorphism.

Sketch of proof. If $g$ is a rational function with poles off $\partial D$, then $S_{g}$ is finite dimensional. Since such $g$ are dense in $C(\partial D), S_{g}$ is compact for all $g \in C(\partial D)$, hence for all $g \in H^{\infty}(\mu)+C(\partial D)$. If $g \in V H^{\infty}(\mu)$ is zero on all but finitely many of the circles in $\partial D$, then $S_{g}$ is compact, in view of the comment after Theorem 8.1. An approximation argument, as at the end of the proof of Theorem 7.1, then shows that $S_{g}$ is compact for any $g \in V H^{\infty}(\mu)$, hence for $g \in V H^{\infty}(\mu)+C(\partial D)$.

The remainder of the proof breaks into three cases. Suppose first there is some boundary circle for which the restriction of $g$ does not belong to $H^{\infty}+C$, say the restriction of $g$ to $\partial \Delta$ is not in $H^{\infty}(d \theta)+C(\partial \Delta)$. By Theorem 8.1, there is then a subspace of $H^{\infty}(d \theta)$ isomorphic to $\ell^{\infty}$ on which the Hankel operator associated with $g$ is an isomorphism. By extending the functions in the subspace to $\Delta$ by the Poisson integral, we obtain a subspace of $H^{\infty}(\mu)$ isomorphic to $\ell^{\infty}$ on which the Hankel operator $S_{g}$ is an isomorphism.

Next suppose that $g$ belongs to $H^{\infty}(\mu)+C(\partial D)$ on each boundary circle, while the limsup of the distances of the restriction of $g$ to $\partial \Delta_{n}$ to $H^{\infty}\left(\mathbb{C}^{*} \backslash \bar{\Delta}_{n}\right)$ is strictly positive. Then there are a subsequence $n_{k} \rightarrow \infty$ and functions $h_{k} \in L^{1}(\mu)$ such that $\left\|h_{k}\right\|_{1}=1, h_{k}=0$ off $\partial \Delta_{n_{k}}$, and $\left|\int h_{k} g d \mu\right| \geq c>0$. We apply the analog of Lemma 5.1, as in the proof of Theorem 7.1, to obtain an embedded copy of $\ell^{\infty}$ on which $S_{g}$ is an isomorphism.

In the remaining case, $g$ belongs to $H^{\infty}+C$ on each boundary circle, and the distance from the restricted $g$ to $H^{\infty}\left(\mathbb{C}^{*} \backslash \bar{\Delta}_{n}\right)$ tends to 0 as $n \rightarrow \infty$. In this case we follow the line of proof of Theorem 7.1 and show that $g \in V H^{\infty}(\mu)+C(\partial D)$. This completes the outline of the proof.

\section{REFERENCES}

[1] M. Behrens, The corona conjecture for a class of infinitely connected domains, Bull. Amer. Math. Soc. 76 (1970), 387-391. MR 41:825

[2] M. Behrens, The maximal ideal space of algebras of bounded analytic functions on infinitely connected domains, Trans. Amer. Math. Soc. 161 (1971), 359-380. MR 55:8380 
[3] J. Bourgain, The Dunford-Pettis property for the ball-algebras, the polydisc-algebras and the Sobolev spaces, Studia Math. 77 (1984), 245-253. MR 85f:46044

[4] J. Bourgain, New Banach space properties of the disc algebra and $H^{\infty}$, Acta Math. 152 (1984), 1-48. MR 85j:46091

[5] J. A. Cima, S. Janson and K. Yale, Completely continuous Hankel operators on $H^{\infty}$ and Bourgain algebras, Proc. Amer. Math. Soc. 105 (1989), 121-125. MR 89g:30065

[6] J. A. Cima, K. Stroethoff and K. Yale, Bourgain algebras on the unit disk, Pacific J. Math. 160 (1993), 27-41. MR 94i:46065

[7] J. A. Cima and R. M. Timoney, The Dunford-Pettis property for certain planar uniform algebras, Michigan Math. J. 34 (1987), 99-104. MR 88e:46023

[8] B. J. Cole and T. W. Gamelin, Tight uniform algebras and algebras of analytic functions, J. Funct. Anal. 46 (1982), 158-220. MR 83h:46065

[9] B. J. Cole and T. W. Gamelin, Weak-star continuous homomorphisms and a decomposition of orthogonal measures, Ann. Inst. Fourier (Grenoble) 35 (1985), 149-189. MR 86m:46051

[10] A. M. Davie, T. W. Gamelin, and J. W. Garnett, Distance estimates and pointwise bounded density, Trans. Amer. Math. Soc. 175 (1973), 37-68. MR 47:2068

[11] T. W. Gamelin, Lectures on $H^{\infty}(D)$, Notas de Matemática, No.21, Universidad Nacional de La Plata, Argentina, 1972.

[12] T. W. Gamelin, Uniform Algebras, 2nd edition, Chelsea Press, 1984. MR 53:14137 (1st ed.)

[13] T. W. Gamelin, Localization of the corona problem, Pacific J. Math. 34 (1970), 73-81. MR 43:2482

[14] T. W. Gamelin, Uniform algebras on plane sets, in Approximation Theory, G.G.Lorentz (ed), Academic Press, 1973, 101-149. MR 49:3548

[15] T. W. Gamelin and J. Garnett, Distinguished homomorphisms and fiber algebras, Amer. J. Math. 92 (1970), 455-474. MR 46:2434

[16] J. Garnett, Bounded Analytic Functions, Academic Press, 1981. MR 83g:30037

[17] P. Gorkin, K. Izuchi and R. Mortini, Bourgain algebras of Douglas algebras, Canad. J. Math. 44 (1992), 797-804. MR 94c:46104

[18] P. Gorkin and Z. Zheng, in preparation.

[19] K. Izuchi, Bourgain algebras of the disk, polydisk, and ball algebras, Duke Math. J. 66 (1992), 503-519. MR 93f: 46082

[20] S. F. Saccone, Banach space properties of strongly tight uniform algebras, Studia Math. 114 (1995), 159-180. MR 96d:46068

[21] L. Zalcman, Bounded analytic functions on domains of infinite connectivity, Trans. Amer. Math. Soc 144 (1969), 241-269. MR 40:5884

Lyman Briggs School, Michigan State University, East Lansing, Michigan 48825

E-mail address: dudziak@pilot.msu.edu

Department of Mathematics, University of California, Los Angeles, California 90024

E-mail address: gamelin@math.ucla.edu

Department of Mathematics, Bucknell University, Lewisburg, Pennsylvania 17837

E-mail address: pgorkin@bucknell.edu 